\title{
MiR-29a and MiR-140 Protect Chondrocytes against the Anti-Proliferation and Cell Matrix Signaling Changes by IL-1 $\beta$
}

\author{
Xianghui Lí, ${ }^{23}$ Zhilei Zhen ${ }^{1,3}$, Guodong Tang ${ }^{1}$, Chong Zheng ${ }^{1}$, and Guofu Yang ${ }^{1, *}$
}

\begin{abstract}
As a degenerative joint disease, osteoarthritis (OA) constitutes a major cause of disability that seriously affects the quality of life of a large population of people worldwide. However, effective treatment that can successfully reverse $O A$ progression is lacking until now. The present study aimed to determine whether two small non-coding RNAs miR-29a and miR-140, which are significantly downregulated in $O A$, can be applied together as potential therapeutic targets for OA treatment. MiRNA synergy score was used to screen the miRNA pairs that potentially synergistically regulate $O A$. An in vitro model of $O A$ was established by treating murine chondrocytes with IL-13. Transfection of miR-29a and miR-140 via plasmids was investigated on chondrocyte proliferation and expression of nine genes such as ADAMTS4, ADAMTS5, ACAN, COL2A1, COL10A1, MMP1, MMP3, MMP13 and TIMP metallopeptidase inhibitor 1 (TIMP1). Western blotting was used to determine the protein expression level of MMP13 and TIMP1, and ELISA was used to detect the content of type II collagen. Combined use of miR-29a and miR-140 successfully reversed the destructive effect of IL-13 on chondrocyte proliferation, and notably affected the MMP13 and TIMP1 gene expression that regulates extracellular matrix. Although co-transfection of miR-29a and miR-140 did not show a synergistic effect on MMP13 protein expression and type II collagen release, but both of them can significantly suppress the protein abundance of MMP13 and restore the type II collagen release in IL-1 $\beta$ treated chondrocytes. Compared with single miRNA transfection, cotransfection of both miRNAs exceedingly abrogated the suppressed the protein production of TIMP1 caused by IL$1 \beta$, thereby suggesting potent synergistic action. These results provided novel insights into the important function of miRNAs' collaboration in OA pathological development.
\end{abstract}

${ }^{1}$ Department of Orthopedics, the First Affiliated Hospital of Harbin Medical University, Harbin, China, ${ }^{2}$ Department of Pharmacy, the Second Affiliated Hospital of Harbin Medical University, Harbin, China, ${ }^{3}$ These authors contributed equally to this work.

*Correspondence: nemoygf@163.com

Received 24 June, 2015; revised 10 October, 2015; accepted 12 October, 2015; published online 25 November, 2015

Keywords: microRNA, miR-29a, miR-140, osteoarthritis
The reduced MMP13, and enhanced TIMP1 protein production and type II collagen release also implies that $\mathbf{m i R}-29 a$ and miR-140 combination treatment may be a possible treatment for $O A$.

\section{INTRODUCTION}

MicroRNAs (miRNAs) are small non-encoding genes, which suppress mRNA expression at the post-transcriptional level (Lewis et al., 2005). Recently, microarray expression analyses revealed significant miRNA dysregulation in osteoarthritis (OA); which strongly implies pathological involvement of these miRNAs (Diaz-Prado et al., 2012; Swingler et al., 2012). Furthermore, by conducting functional experiments, miRNAs are suggested to be implicated in $O A$, and significantly have crucial functions in OA-specific pathology regulation, such as chondrogenesis (Le et al., 2013). Recently, in addition to the cartilagespecific miRNA miR-140 (Miyaki et al., 2010), the other miRNAs, such as miR-29a (Guerit et al., 2014), miR-26a (Etich et al., 2015), miR-146 (Yamasaki et al., 2009), and miR-149 (Santini et al., 2014), etc have been also validated of their important functions in cartilage function repairs (Matsukawa et al., 2013; Park et al., 2013; Song et al., 2013). As suggested by system biology approaches (Xu et al., 2011; Zhu et al., 2013), joint participation of multiple miRNAs in regulating the same pathological process induces the potential collaboration of different miRNAs. We proposed that the collaborative or synergistic potential shown by different miRNAs may suggest a novel treatment modality, in which the process of complex disease can be reversed by simultaneous intervention of multiple endogenous biological factors (such as miRNAs), instead of exogenous chemical drugs as Small and Olson discussed (Small and Olson, 2011).

Despite that synergistic miRNA actions have been widely studied in cancer and cardiovascular disease (Bandi and Vassella, 2011; Dong et al., 2012; Hu et al., 2011; Noguchi et al., 2013; Park et al., 2009; Pencheva et al., 2012), no such evidence is found in OA. Based on the broad participation of miRNAs in OA (Matsukawa et al., 2013; Miyaki et al., 2010; Park et al., 2013; Song et al., 2013), we supposed that different miRNAs may co-regulate OA-related genes and synergistically affect the associated pathological processes. The current study is the first to investigate the synergistic actions of two miRNAs (miR-29a and miR-140) in an in vitro model of interleukin 1-beta 
(IL-1 $\beta$ )-treated chondrocytes. MiR-140 is generally recognized as cartilage-specific miRNA, which has chief functions in OA pathogenesis (Miyaki and Asahara, 2012; Zhang et al., 2013). MiR-29a was also detected to undergo differential expression in OA (Swingler et al., 2012), which is an important regulator of collagen expression in related human disease (Kwiecinski et al., 2011; Maurer et al., 2010; Wang et al., 2012). Our results revealed that miR-29a and miR-140 can significantly reverse the effect of IL-1 $\beta$ pre-treatment on chondrocytes, by significantly influencing matrix metallopeptidase 13 (MMP13) and TIMP metallopeptidase inhibitor 1 (TIMP1) expression in both mRNA and protein levels, and subsequently affecting the content of type II collagen in chondrocytes. A synergistic restoration of TIMP1 was shown in IL-1 $\beta$ pre-treatment chondrocytes transfected with combination miRNAs compared with single transfection, in which over-expression should be responsible for $O A$ progression (Hsieh et al., 2013; Wang et al., 2013). In summary, our findings provided the earliest evidence regarding the synergistic effects of miRNAs in OA.

\section{MATERIALS AND METHODS}

\section{Synergy score of miRNA pairs}

MiRNA synergy score has been applied to determining whether two given miRNAs tend to act synergistically according to the method of Zhu et al. (2013). Briefly, it comprises two independent parameters, the target similarity score (TSS) and the protein interaction score (PIS). TSS and PIS quantitatively measure the degree of gene co-regulation by two miRNAs and functional association between their target gene products, respectively. Synergy score $=$ TSS $+\alpha \cdot$ PIS, $\alpha$ is a correction factor for the contribution of PIS to synergy score. According to the ROC curve of synergy score in distinguishing between the high and low co-regulation groups of miRNA pairs, when the correction factor $\alpha$ is set at 1.8 , synergy score possesses the optimal performance in distinguishing between the high and low coregulation groups of miRNA pairs.

\section{Murine chondrocyte preparation}

The murine chondrocytes were isolated with the methods described previously (Gosset et al., 2008; Salvat et al., 2005). Briefly, immature mouse articular chondrocytes, which were derived from three-day-old sucking mice, were grown at $37^{\circ} \mathrm{C}$ in Dulbecco's modified Eagle medium (DMEM) (GIBCO, USA) supplemented with $10 \%$ fetal bovine serum (FBS). The medium was changed every other day. The use of animals was approved by the Experimental Animal Ethic Committee of the Harbin Medical University, China (Animal Experimental Ethical Inspection Protocol, No. 2009104). Afterward, the cells cultured at $70 \%$ to $80 \%$ confluence were divided into normal group and $\mathrm{IL}-1 \beta$ induction group. The medium for the normal group was replaced with DMEM supplemented with $1 \%$ FBS; and the medium for the IL-1 $\beta$ induction group was replaced with DMEM

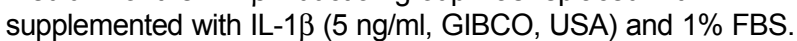
Five $\mathrm{ng} / \mathrm{ml} \mathrm{IL}-1 \beta$ causes significant morphological changes of immature mouse articular chondrocytes (data not shown). Abnormal cell morphology might indicate degradation and loss of normal cell function of chondrocytes (Murray et al., 2010). These two kinds of cells were used in the following experiments.

\section{Plasmid construction and transfection}

The specific pre-miRNA single strand DNA oligos were designed and synthesized for miR-26a, miR-29a, miR-140 and miR-146. Subsequently, the DNA oligos were annealed and ligated with $\mathrm{pEGFP-C1}$ plasmids. Double-digestion with restric- tion enzyme and DNA sequencing were used to verify the plasmids. Consequently, the plasmids were extracted and purified, using an endotoxin-free plasmid extraction kit (EndoFree Plasmid Maxi Kit, QIAGEN) from transformed Escherichia coli. Afterward, the purified plasmids were dissolved in TE buffer (10 $\mathrm{mM}$ Tris- $\mathrm{HCl}$ and $1 \mathrm{mM}$ EDTA, $\mathrm{pH}$ 8.0), and transfected into chondrocytes with Lipofectamine 2000 transfection reagent (Invitrogen), following the manufacturer's instructions. A random sequence annealed with pEGFP-C1 plasmid serves as negative control (NC). MiRNAs and NC were transfected to the chondrocytes as the indicated concentrations, for example, 20 $\mathrm{nM}$ miRNA or/and $0.125 \mu \mathrm{g}$ plasmid DNA, $40 \mathrm{nM}$ miRNA or/and $0.25 \mu \mathrm{g}$ plasmid, $60 \mathrm{nM}$ miRNA or/and $0.375 \mu \mathrm{g}$ plasmid DNA, etc. The efficiency of the transfection was confirmed by quantitative real-time reverse transcription-polymerase chain reaction (qRT-PCR).

\section{Proliferation test}

Cell proliferation was measured with $\mathrm{CCK}-8$ at different time points. Briefly, first generation of chondrocytes (IL-1 $\beta$-induced chondrocytes and IL-1 $\beta$-induced chondrocytes transfected with plasmids of miR-29a, miR-140, miR-29a and miR-140) were harvested and adjusted to $5 \times 10^{4}$ cells $/ \mathrm{ml}$ with DMEM. Afterward, $100 \mu \mathrm{l}$ of different cells were added to the wells of a 96well plate in quintuplicate, and were inoculated at $37^{\circ} \mathrm{C}$ in a $5 \%$ $\mathrm{CO}_{2}$ incubator for $1,2,4,6$, and 8 days. After incubation, $90 \mu \mathrm{l}$ DMEM and $10 \mu \mathrm{l}$ CCK-8 solution was added to each well and incubated for another $4 \mathrm{~h}$. The optical density at $450 \mathrm{~nm}$ was then measured using a microplate reader.

\section{Immunofluorescence staining}

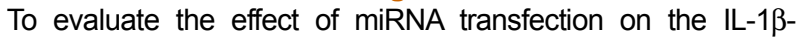
induced chondrocyte proliferation and the Ki-67 expression, which is a kind of nuclear proliferation-associated antigen, was examined using indirect immunofluorescence staining. Briefly, cells were fixed with $4 \%$ paraformaldehyde for $30 \mathrm{~min}$. To quench paraformaldehyde, the slides were immersed in $50 \mathrm{mM}$ $\mathrm{NH}_{4} \mathrm{Cl}$ for $10 \mathrm{~min}$. Afterward, the cells were washed with PBS, which was permeabilized with $0.1 \%$ Triton X-100 in PBS for 15 min, and then blocked with $10 \%$ BSA in PBS for 30 min. After incubation with anti-Ki-67 (Santa Cruz, sc-7846) or PBS at $4^{\circ} \mathrm{C}$ overnight, the cells were washed and incubated with Alexa Fluor $^{\circledR} 647$ donkey anti-goat $\operatorname{lgG}(\mathrm{H}+\mathrm{L})(1: 200$, Invitrogen, A21447) for $30 \mathrm{~min}$. Subsequently, the cells were stained with DAPI for $30 \mathrm{~min}$; and the slides were mounted and examined under a fluorescence microscope.

\section{Quantitative real time-PCR ( $q R T-P C R)$ analysis}

Total RNA was extracted using RNeasy FibrousTissue mini kit (QIAGEN) according to the manufacturer's guide, and was reverse-transcribed by random hexamer primer. Afterward, RTPCR analysis was performed using the GeneAmp 7500 system (Applied Bioscience) and SYBR ${ }^{\circledR}$ Green (Applied Bioscience). All expression data were normalized to $\beta$-actin. Each reaction contained $1 \mu \mathrm{l} 20 \times$ SYBR Green, $1 \mu \mathrm{l}$ sense and antisense specific primer, and $1 \mu \mathrm{l}$ cDNA matrix, in a $20 \mu \mathrm{l}$ final volume. All reactions were run in duplicate. PCR products obtained after 2 min at $95^{\circ} \mathrm{C}$, followed by 40 cycles of melting for $10 \mathrm{~s}$ at $95^{\circ} \mathrm{C}$, $30 \mathrm{~s}$ at $60^{\circ} \mathrm{C}$, and $30 \mathrm{~s}$ at $70^{\circ} \mathrm{C}\left(70^{\circ} \mathrm{C}\right.$ to $\left.95^{\circ} \mathrm{C}\right)$. The primers used for amplification were as follows: $\beta$-actin (Forward: 5 'ggttgtctcctgcgacttca-3'; Reverse: 5'-tagggcctctcttgctcagt-3'), ADAMTS4 (Forward: 5'-aggccggaaataacctcact-3'; Reverse: 5'gggtagagggggcagtttag-3'), ADAMTS5 (Forward: 5'-tgcccacccaa tggtaaatct-3'; Reverse: 5'-ttggaccagggcttggatg-3'), COL2A1 (Forward: 5'-gccaagacctgaaactctgc-3'; Reverse: 5'-gccatagctga 
Table 1. The synergistic effects of miRNA pairs

\begin{tabular}{llll}
\hline miRNA pairs & TSS & PIS & SS \\
\hline miR-140 : miR-29a & 0.143 & 0.357 & 0.786 \\
miR-26a : miR-29a & 0.063 & 0.375 & 0.738 \\
miR-140 : miR-26a & 0 & 0.4 & 0.720 \\
miR-140 : miR-146 & 0.364 & 0.091 & 0.528 \\
miR-146 : miR-29a & 0.063 & 0.25 & 0.513 \\
miR-147 : miR-9 & 0.267 & 0.067 & 0.388 \\
miR-98 : miR-147 & 0.231 & 0.077 & 0.370 \\
miR-98: miR-9 & 0.2 & 0.067 & 0.321 \\
miR-26a : miR-146 & 0.067 & 0.133 & 0.306 \\
miR-16 : miR-9 & 0.25 & 0.250 \\
miR-16 : miR-98 & 0.25 & 0 & 0.250 \\
miR-16 : miR-147 & 0.083 & 0 & 0.232 \\
\hline
\end{tabular}

TSS, target similarity score; PIS, protein interaction score; SS, synergy score

agtggaagc-3'), COL10A1 (Forward: 5'-gcagcattacgacccaagat3'; Reverse: 5'-tctgtgagctccatgattgc-3'), ACAN (Forward: 5'ggcagtggagagtcttctgg-3'; Reverse: 5'-ctgctcccagtctcaactcc-3'), MMP1 (Forward: 5'-gtgctctccttccacagagg-3'; Reverse: 5'ggtccacgtctcatcaaggt-3'), MMP3 (Forward: 5'-tggagatgctcactttg acg-3'; Reverse: 5'-gccttggctgagtggtagag-3'), MMP13 (Forward: 5'-cttctggcacacgctttcc-3'; Reverse: 5'-tcttcatcgcctggaccata-3') and TIMP1 (Forward: 5'-agataccatgatggccccct-3'; Reverse: 5'cgctggtataaggtggtctcg-3'). Relative quantity of product was expressed as fold-induction of the target gene compared with the control primers according to the formula $2-\Delta \Delta C T$.

\section{Western blot analysis}

To detect MMP13 and TIMP1 expression, chondrocytes were lysed in RIPA lysis buffer (1\% TritonX-100, 1\% deoxycholate, $0.1 \%$ PMSF, and $0.1 \%$ SDS). Protein concentrations were determined using the Bradford protein assay kit (Beyotime Institute of Biotechnology). After boiling for $5 \mathrm{~min}$, the lysates were separated on $10 \%$ SDS-PAGE polyacrylamide gel. Proteins were transferred to PVDF membranes, which were blocked in Tris-buffered saline with Tween-20 (TTBS) containing 5\% non-fat dried milk. The membranes were incubated with primary antibody against MMP13 (USA, 1: 200 dilution), TIMP1 (USA, 1:200 dilution), and $\beta$-actin (Kangcheng, China, 1: 500 dilution) at $4^{\circ} \mathrm{C}$ overnight. After rinsing thrice in TTBS, the membranes were incubated with secondary antibody conjugated with horseradish peroxidase at room temperature for $1 \mathrm{~h}$, and were then developed using a BeyoECL Plus chemiluminescence detection kit (Beyotime Institute of Biotechnology), according to the manufacturer's guide. The membranes were subsequently exposed to X-ray film. The relative expression levels of these proteins were determined through densitometric scanning and by calculating the ratios of each protein to $\beta$-actin bands, which were expressed constitutively.

\section{Enzyme-linked immunosorbant assay (ELISA)}

The expression of type II collagen was also investigated by ELISA using the type II collagen detection kit (Chondrex Inc. USA) according to themanufacturer's instructions. Briefly, collagen was digested by $10 \mathrm{mg} / \mathrm{ml}$ pepsin dissolved in $0.05 \mathrm{M}$
$\boldsymbol{A}$

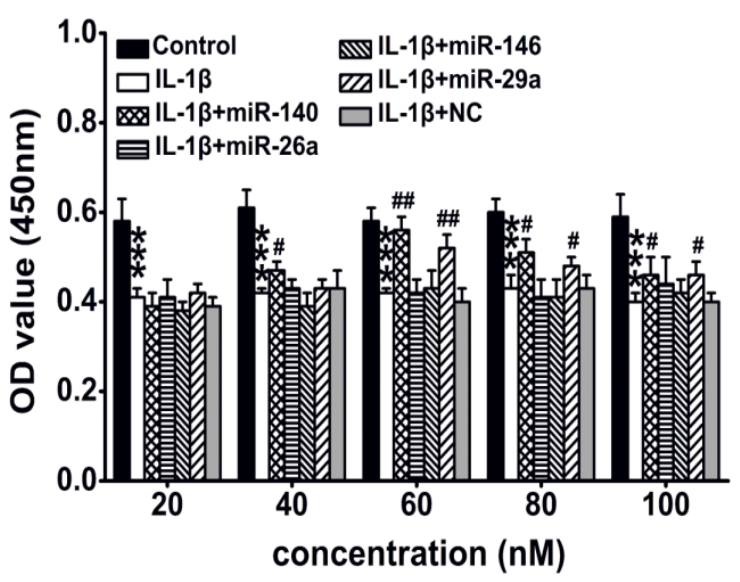

B

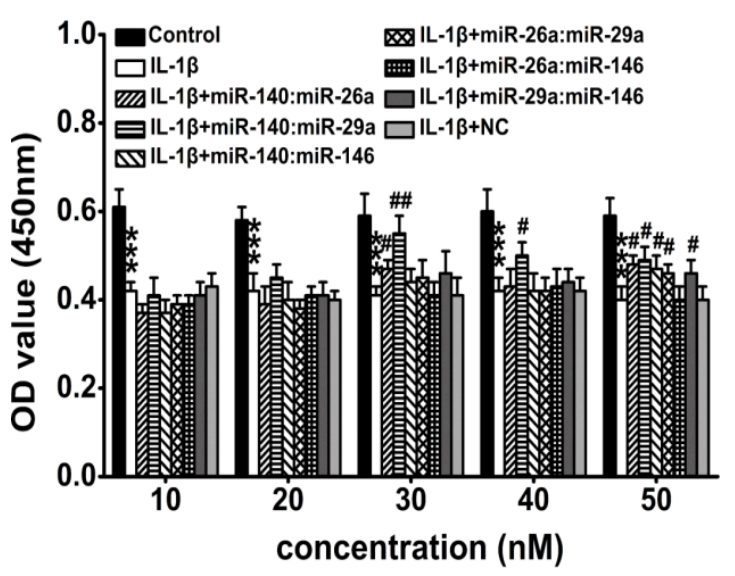

Fig. 1. Screening of miRNA pairs that regulating cell proliferation in IL-1 $\beta$ treated chondrocytes. (A) Effect of single miRNA transfection on cell proliferation. (B) Effect of miRNA pairs on cell proliferation. Two miRNA with a ratio of 1:1 were transfected to chondrocytes. Cell proliferation was detected six days after administration of IL-1 $\beta .{ }^{* * *} p<0.001$ vs control; ${ }^{\# \text {, }}$ respectively indicates $p<0.05, p<$ 0.01 vs IL-1 $\beta ; n=6 / g r o u p$. 
acetic acid overnight at $4^{\circ} \mathrm{C}$ with gentle mixing. Cells were then incubated at $37^{\circ} \mathrm{C}$ for $30 \mathrm{~min}$ with $1 \mathrm{mg} / \mathrm{ml}$ pancreatic elastase, after that samples were centrifuged and supernatants were diluted with the standard dilution buffer provided by the kit. The ELISA plate was coated with collagen type II antibody as described by the supplier; $100 \mathrm{ml} /$ well of sample was added and incubated for $2 \mathrm{~h}$ at room temperature. After washing, samples were incubated with streptavidin peroxidaselabeled detection reagent (dilution 1: 200) for $1 \mathrm{~h}$ at room tem- perature. Finally, after rinsing plates, color reaction followed through $\mathrm{H}_{2} \mathrm{O}_{2}$ incubation for $30 \mathrm{~min}$ at room temperature, reaction was stopped with $100 \mu \mathrm{l} /$ well of $2.5 \mathrm{~N}$ sulfuric acid. The OD was read at $490 \mathrm{~nm}$ in an ELISA reader (TECAN, Switzerland).

\section{Statistical analysis}

The data are expressed as mean \pm SEM from at least three independent experiments. Statistical analysis was performed
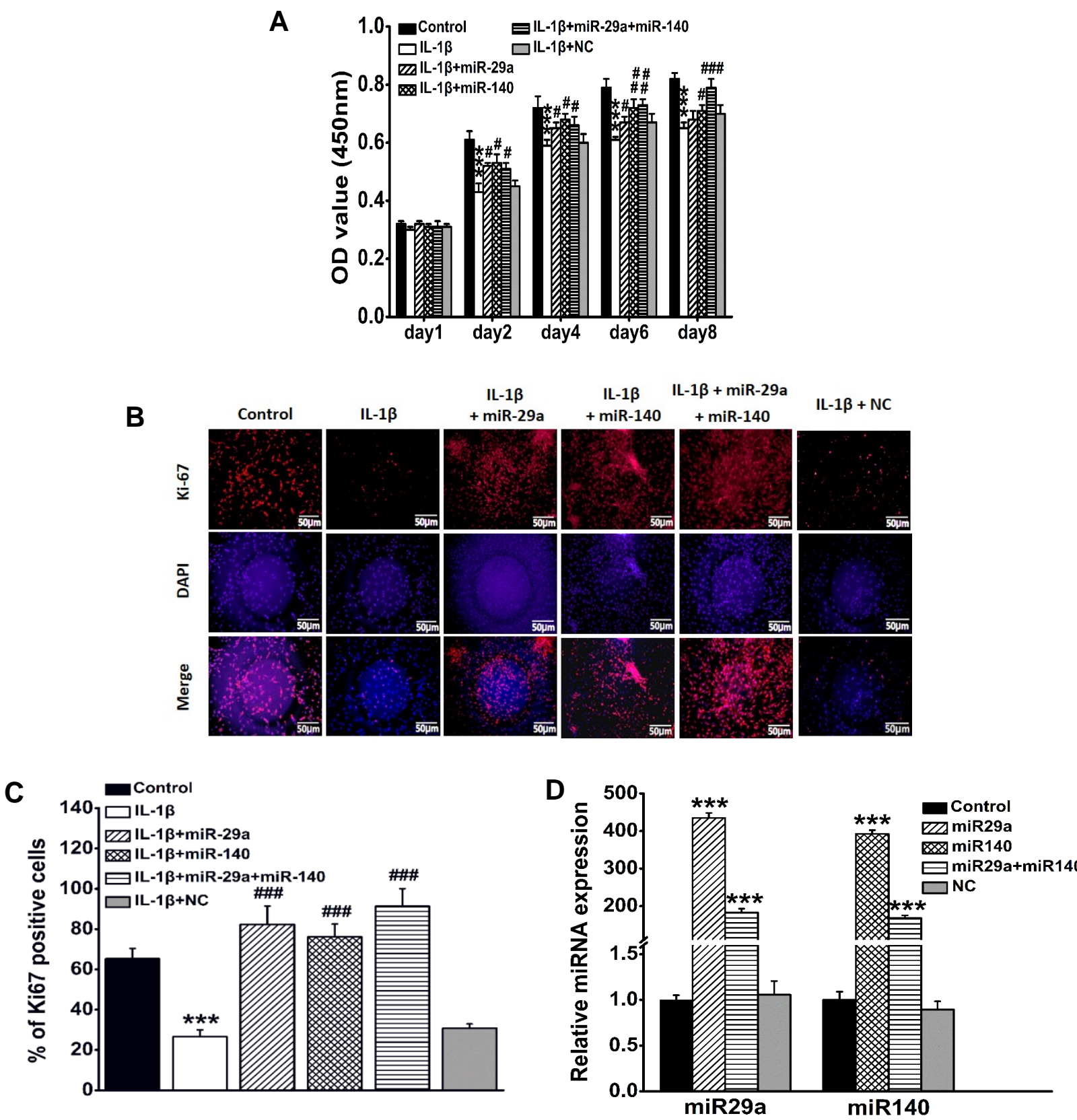

Fig. 2. miR-29a and miR-140 reversed IL-1 $\beta$-mediated inhibition of chondrocytes proliferation. (A) Cell proliferation by CCK8 assay ( $\mathrm{n}=5$ ). $* * *$ indicates $p<0.001$ vs control; \#, "\#, respectively indicates $p<0.05, p<0.01, p<0.001$ vs IL-1 3 . (B) Cell proliferation by Ki-67 immunofluorescence staining. (C) Statistical bar graph of (B). Ki-67-positive cells were counted from 10 images obtained in randomly selected fields from each group. ${ }^{* *}$ indicates $p<0.001$ vs control; ${ }^{\ldots \prime \prime}$ indicates $p<0.001$ vs IL-1 $\beta$. (D) Relative miRNA expression level after transfection determined by qRT-PCR. ${ }^{* \star *}$ indicates $p<0.001$ vs control. 
with one-way or two-way ANOVA, followed by Tukey's test for multiple comparisons. Differences were only considered to be significant at $p<0.05$.

\section{RESULTS}

Synergistic cytoprotection of miRNA pairs against IL-1 $\beta$ induced decrease of cell proliferation in primary cultured chondrocytes

We constructed the synergistic miRNA network on OA, miRNA pair synergy score was calculated and shown in Table 1. Table 1 showed the top 12 miRNA pairs which have potential synergistic regulation role in OA. Among these miRNA pairs, miR140/miR-29a showed the highest synergy score (0.786). The miRNA pairs with top five synergy score were examined the synergistic cytoprotection in IL-1 $\beta$-treated chondrocytes. First we detected the effect of single miRNA transfection on chondrocytes proliferation that treated with $5 \mathrm{ng} / \mathrm{ml} \mathrm{IL-1 \beta}$, and concentration miRNA ranging from $20 \mathrm{nM}$ to $100 \mathrm{nM}$. As shown in Fig. 1A, miR-140 and miR-29a significantly reversed the decreased cell proliferation induced by IL-1 $\beta$, and $60 \mathrm{nM}$ miR-140 or miR-29a showed the most significant anti-proliferation role in IL-1 $\beta$ treated chondrocytes. Then we detected co-transfection of miRNA pairs (1:1) with one miRNA dosage ranging from 10 to $50 \mathrm{nM}$. Our results showed that a moderate preservation of cell proliferation afforded by mono-miRNA transfection was seen only at a higher concentration (60-100 nM). By comparison, as shown in Fig. 1B apparently a higher relative cell proliferation was obtained by pair-wise transfection of miRNAs (miR140/miR29a: $30 \mathrm{nM} / 30 \mathrm{nM}$ ). These results suggest that miRNA pair (miR140/miR29a) may have the potential to protect IL-1 $\beta$-induced injury in primary cultured chondrocytes.

\section{MiR-29a and miR-140 protected chondrocytes against IL-1 $\beta$ anti-proliferation}

In order to confirm the role miRNA pair of miR-29a and miR-140 in the cell proliferation in IL-1 $\beta$-treated chondrocytes, we compared the effect of miRNA pair and single miRNA transfection on cell proliferation by two methods, CCK-8 assay and Ki-67 staining. The CCK8 assay result revealed that cells incubated with IL-1 $\beta$ underwent a slow cell proliferation process for eight days, compared with the cultured chondrocytes without IL-1 $\beta$. Transfection of miR-29a $(60 \mathrm{nM}), \mathrm{miR}-140(60 \mathrm{nM})$, or both $(30 \mathrm{nM} / 30 \mathrm{nM})$ can partly reverse the IL-1 $\beta$ anti-proliferative effect two days after transfection, and the effect of co-transfection of miR-29a and miR-140 was time-dependent and reach a peak eight days after transfection (Fig. 2A). This finding was further verified via immunofluorescence. Only after two days, Ki-67 over-expression, which is cell proliferation associated nuclear antigen, appeared in chondrocytes that were transfected with miR-29a, miR-140,
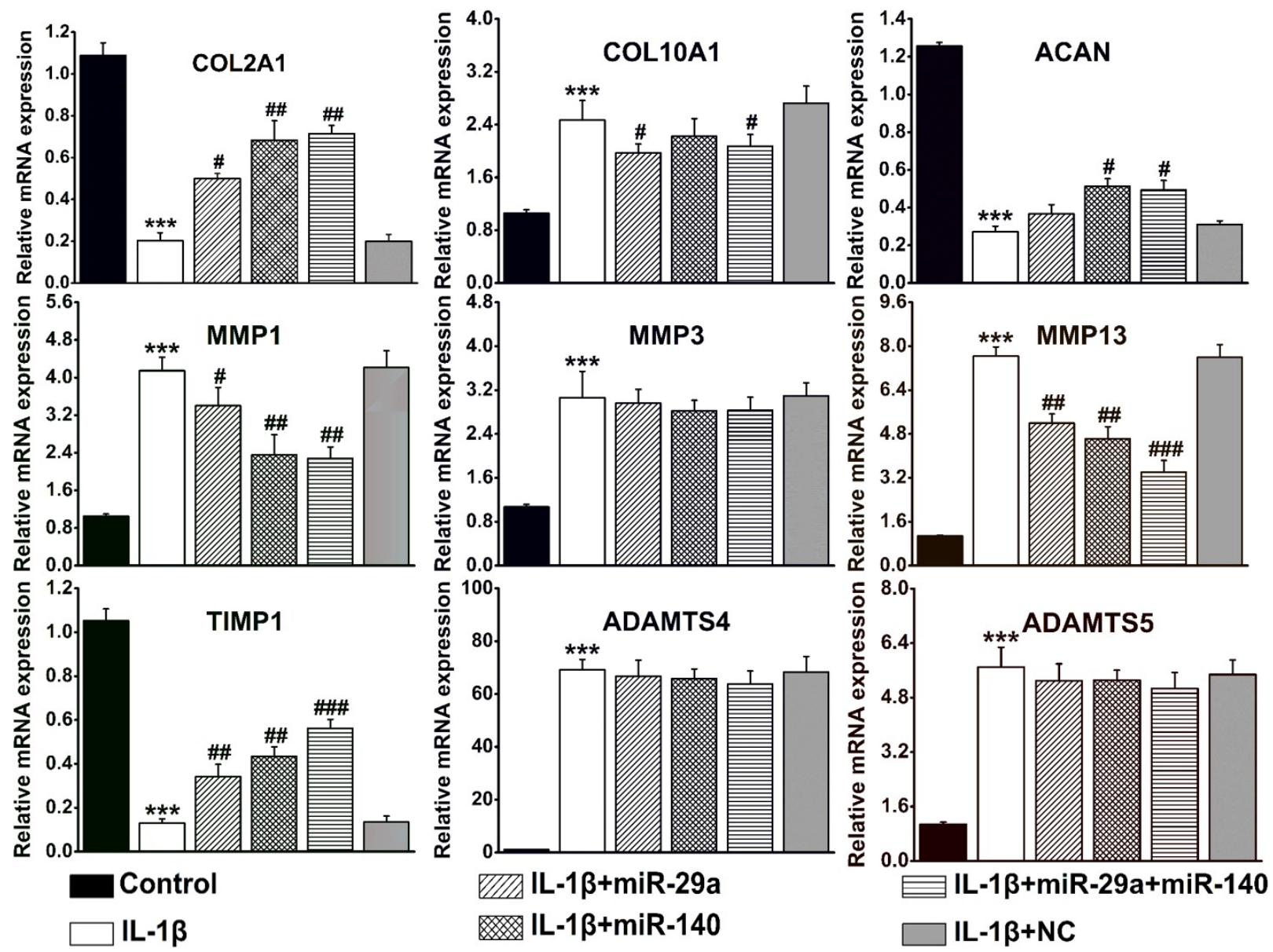

Fig. 3. Quantitative RT-PCR assays the mRNA expression of COL2A1, COL10A1, ACAN, MMP1, MMP3, MMP13, TIMP1, ADAMTS4 and ADAMTS5. ${ }^{* \star *}$ indicates $p<0.001$ vs control;,$"$ "\#" respectively indicates $p<0.05, p<0.01$ and $p<0.001$ vs IL-1 $1 ; ; \mathrm{n}=3$. 
or both (data not shown). Notably, a stable effect on promoting cell proliferation was investigated for miR-29a and miR-140 with regard to the prolongation of cell culture time. Ki-67 staining eight days after transfection showed that single miRNA and miRNA pair transfection significantly reversed the inhibited cell proliferation by IL-1 $\beta$ (Figs. $2 \mathrm{~B}$ and $2 \mathrm{C}$ ). qRT-PCR was used to confirm the transfection efficiency eight days after transfection, and the results showed that the relative miRNA level of miR29a and miR-140 were $434.87 \pm 13.40$ and $392.55 \pm 9.94$ fold, respectively; they were $183.21 \pm 9.90$ and $167.67 \pm 7.59$ fold respectively in simultaneously transfection of both miRNAs (Fig. 2D). These results suggest that combination of miR-29a and miR-140 protects chondrocytes against the decreased cell proliferation caused by IL-1 $\beta$.

\section{Screening of miR-29a and miR-140 on the genes that regulating cell matrix signaling}

The ECM of cartilage tissue consists of collagen type II, other collagens and noncolla-genous structural proteins. In physiological conditions the ECM is extremely stable. But in OA, cell matrix signaling is disrupted by depletion of collagen and proteoglycan due to the production of MMPs, ADAMTSs and other proteases, resulting in matrix degradation and mechanical loss of tissue resilience matrix proteinases. Here, we analyzed the genes encode collagens COL2A1, COL10A1 and ACAN, the ACAN gene encodes an important structural component of ECM, namely, aggrecan (Lark et al., 1997), the genes that encode degradative enzymes MMP1, MMP3, MMP13, ADAMTS 4 , or ADAMTS5, and TIMP1 gene which is an inhibitor of the
MMPs. As shown in Fig. 3, after administration of IL-1 $\beta$ for two days, the mRNA expression levels of COL2A1 and ACAN were significantly decreased. The mRNA level of COL10A1 which is related to chondrocyte hypertrophy was significantly induced by IL-1 $\beta$. The mRNA expression levels of MMP1, MMP3, MMP13, ADAMTS4, ADAMTS5 were up-regulated by IL-1 $\beta$, and the mRNA expression level of TIMP1 was down-regulated by IL-1 $\beta$ (Fig. 3). However, the miR-29a transfection can obviously alter the mRNA expression of COL2A1, COL10A1, MMP1, MMP13 and TIMP1 induced by IL-1 13 , miR-140 transfection can significantly affect the mRNA expression of COL2A1, ACAN, MMP1, MMP13 and TIMP1 induced by IL-1 $\beta$. Although ADAMTS5 has been suggested a potent target of miR-140, but neither miR140 nor miR-29a transfection failed to show any impact on the expression mRNA of ADAMTS5 and ADAMTS4. Nonetheless, we interestingly found that co-transfection of miR-29a and miR140 further increased the mRNA expression of and TIMP1, decreased the mRNA expression of MMP13 compared with that of miR-140 (Fig. 3). These results indicate that miR-29a and miR-140 may affect the ECM abundance by regulating the mRNA expression of MMP13 and TIMP1.

\section{MiR-29a and miR-140 synergistically suppressed cell matrix signaling through regulating the TIMP1and MMP13 protein level and the release of type II collagen}

To further confirm the effect of both miR-29a and miR-140 on IL-1 $\beta$-induced ECM production of murine chondrocytes. Western blotting was used to determine the expression level of MMP13 and TIMP1 protein (Fig. 4). Transfection of miR-29a or
$\boldsymbol{A}$

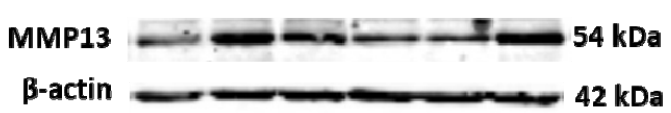

B

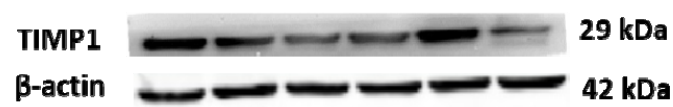

C
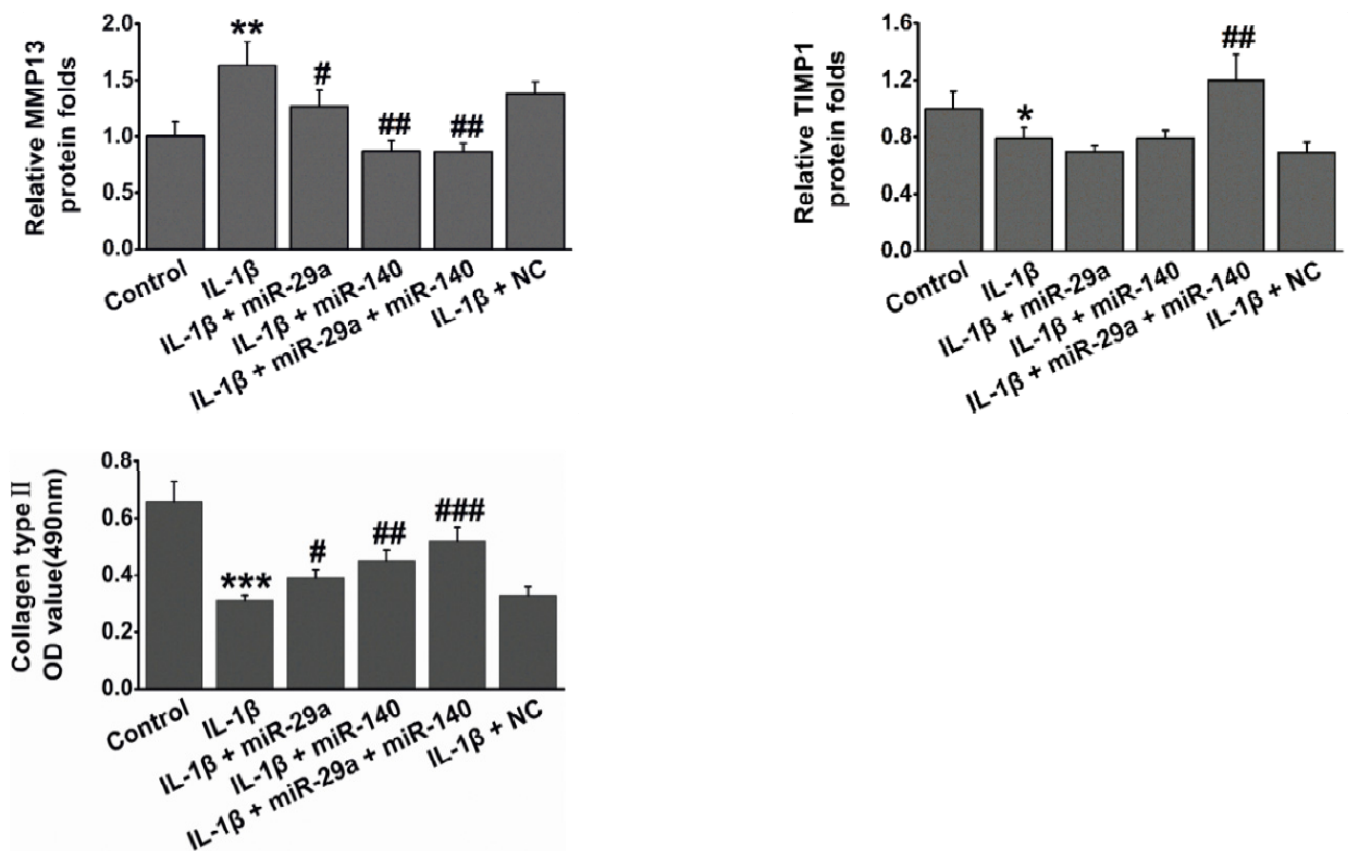

Fig. 4. Effect of miR-29a and miR-140 on MMP13, TIPM1 and type II collagen in IL-1 $\beta$-treated chondrocytes. Protein expression of MMP13 (A) and TIMP1 (B) were determined by Western blot, and the content of type II collagen (C) was determined by ELISA. *, ** respectively indicates $p<0.05$ and $p<0.01$ vs control; "\#, respectively indicates $p<0.05, p<0.01$ and $p<0.001 \mathrm{vs}$ IL-1 1 ; $\mathrm{n}=4$. 
miR-140 alone significantly decreased MMP13 protein production compared with that in IL-1 $\beta$-treatment group. However, simultaneously transfected with miR-29a and miR-140 did not show a synergistic effect on suppressing the MMP13 protein production (Fig. 4A). Transfection of miR-29a or miR-140 alone did not reverse the inhibition of TIMP1 caused by IL-1 $\beta$ treatment, but both miRNAs together were able to significantly abrogate the inhibitory effect of IL-1 $\beta$ on TIMP1 (Fig. 4B). The greater protein abundance of TIMP1 can only be obtained through co-transfection of miR-29a and miR-140, which strongly suggests a synergistic action of them. MMP13 and TIMP1 are crucial for the degradation of type II collagen during OA, therefore we determined the content of type II collagen in the chondrocytes which were transfected with single miR-29a or miR-140, or both. The results showed that combination transfection of miR-29a and miR-140 significantly restored the reduced level of type II collagen caused by IL-1 $\beta$ (Fig. 4C). Taken together, miR-29a and miR-140 combination treatment may be a hopeful therapeutic avenue for OA.

\section{DISCUSSION}

As the population worldwide ages, OA, which is the most common type of arthritis, is gradually becoming a serious health problem for individuals and a huge economic burden for the whole social health system. Currently, merely symptom management is accessible for patients with OA. Failure to reverse the disease process implies that a long-term disease management and continued medical costs are definitely necessary.

Just recently, the ongoing reversal of the "macro" roles of miRNAs sheds novel insights into the pathological mechanisms underlying OA (Le et al., 2013; Miyaki and Asahara, 2012). Great body of evidence all suggests that miRNAs might possess hopeful potential as therapeutic targets for OA treatment in the future (Matsukawa et al., 2013; Miyaki et al., 2010; Park et al., 2013; Song et al., 2013). After careful consideration of some technical issues, Small and Olson suggested that miRNAs may be more feasible and advantageous for drug intervention when compared with other classes of biological factors (Small and Olson, 2011). With the support of mature designs of drug delivery system, we can even consider simultaneous intervention of multiple miRNAs, by which synergistic effects of miRNAs can be activated, and therapeutic selectivity can be obtained with lower doses and fewer side effects (Lehar et al., 2009). However, when encountered with numerous differentially expressed genes in OA (Diaz-Prado et al., 2012; Swingler et al., 2012), making a reasonable decision that miRNAs should be combined for therapeutic consideration is difficult. By applying the system biology approach developed by Zhu et al. (2013), we examined and dissected the interactomics of target genes of OA-related miRNAs in previous work, and identified that miR29a and miR-140 might be synergistic in posttranscriptional gene regulation (data not shown). Thus, the aim of this study is to further validate, in vitro, the synergistic effect of miR-29a and miR-140 on IL-1 $\beta$-induced chondrocytes in the laboratory. More importantly, such effortful clinical implications can be provided, whether these implications can make more sense by combination of multiple miRNA application in potential therapeutics for OA

Compared with transfection of miR-140 alone, apparent advantage cannot be found for the combined use of miR-140 with miR-29a against the anti-proliferative effect of IL-1 $\beta$. However, synergistic benefits brought by these genes still appeared in dramatically decreasing mRNA expression of MMP13, which encodes a protease to degrade type IV collagen; and increasing mRNA expression of TIMP1, which encodes a metallopep- tidase inhibitor; by which the two miRNAs may restore the ECM in cartilage tissue. More important evidence is that miR-29a and miR-140 co-transfection synergistically up-regulated TIMP1 protein expression in IL-1 $\beta$-treated chondrocytes. TIMP1 is pathologically related with $\mathrm{OA}$ and functional experiments verified that this protein has important regulatory functions in $\mathrm{OA}$ progression (Hsieh et al., 2013; Wang et al., 2013). In addition to its inhibitory role against most of the known MMPs, TIMP1 is able to promote cell proliferation in a wide range of cell types, and may also have an anti-apoptotic function. Therefore, the restoration of TIMP1 caused by miR-29a and miR-140 may explain their mediating on ECM production and cell proliferation in the chondrocytes. Overall, we supposed that the synergy showed by miR-29a and miR-140 may promote cell proliferation of the insulted chondrocytes with more additional benefits, such as extracellular environment improvement.

In conclusion, our study provided the direct experimental evidence for synergistic effect of OA-related miRNAs. However, more functional in vivo experiments are definitely needed to validate the effect of miR-29a and miR-140 co-administration. In addition, results strongly suggest that intervention of multiple synergistic miRNAs may provide more comprehensive and pathology-selective protection for the injured cartilage cells. Our effort provides implications for multiple miRNA treatment for OA in the future.

\section{ACKNOWLEDGMENTS}

This work was supported by the National Natural Science Foundation of China (grant 81250033), the Heilongjiang Postdoctoral Grant (grant LBH-Q13122) and the Doctor Foundation of the First Affiliated Hospital of Harbin Medical University (grant 2011BS016). Study sponsors had no involvement in study design, collection, analysis and interpretation of data, the writing of the manuscript or in the decision to submit the manuscript for publication.

\section{REFERENCES}

Bandi, N., and Vassella, E. (2011). miR-34a and miR-15a/16 are coregulated in non-small cell lung cancer and control cell cycle progression in a synergistic and $\mathrm{Rb}$-dependent manner. Mol. Cancer 10, 55.

Diaz-Prado, S., Cicione, C., Muinos-Lopez, E., Hermida-Gomez, T., Oreiro, N., Fernandez-Lopez, C., and Blanco, F.J. (2012). Characterization of microRNA expression profiles in normal and osteoarthritic human chondrocytes. BMC Musculoskelet Disord. 13, 144.

Dong, C.G., Wu, W.K., Feng, S.Y., Wang, X.J., Shao, J.F., and Qiao, J. (2012). Co-inhibition of microRNA-10b and microRNA-21 exerts synergistic inhibition on the proliferation and invasion of human glioma cells. Int. J. Oncol. 41, 1005-1012.

Etich, J., Holzer, T., Pitzler, L., Bluhm, B., and Brachvogel, B. (2015) MiR-26a modulates extracellular matrix homeostasis in cartilage. Matrix Biol. 43, 27-34

Gosset, M., Berenbaum, F., Thirion, S., and Jacques, C. (2008) Primary culture and phenotyping of murine chondrocytes. Nat. Protoc. 3, 1253-1260

Guerit, D., Brondello, J.M., Chuchana, P., Philipot, D., Toupet, K., Bony, C., Jorgensen, C., and Noel, D. (2014). FOXO3A regulation by miRNA-29a Controls chondrogenic differentiation of mesenchymal stem cells and cartilage formation. Stem Cells Dev. 23, 1195-1205.

Hsieh, J.L., Shiau, A.L., Lee, C.H., Yang, S.J., Lee, B.O., Jou, I.M., Wu, C.L., Chen, S.H., and Shen, P.C. (2013). CD8+ T cellinduced expression of tissue inhibitor of metalloproteinses-1 exacerbated osteoarthritis. Int. J. Mol. Sci. 14, 19951-19970.

Hu, S., Huang, M., Nguyen, P.K., Gong, Y., Li, Z., Jia, F., Lan, F., Liu, J., Nag, D., Robbins, R.C., et al. (2011). Novel microRNA prosurvival cocktail for improving engraftment and function of 
cardiac progenitor cell transplantation. Circulation 124, S27-34.

Kwiecinski, M., Noetel, A., Elfimova, N., Trebicka, J., Schievenbusch, S., Strack, I., Molnar, L., von Brandenstein, M., Tox, U., Nischt, R., et al. (2011). Hepatocyte growth factor (HGF) inhibits collagen I and IV synthesis in hepatic stellate cells by miRNA-29 induction. PLoS One 6, e24568.

Lark, M.W., Bayne, E.K., Flanagan, J., Harper, C.F., Hoerrner, L.A., Hutchinson, N.I., Singer, II, Donatelli, S.A., Weidner, J.R., Williams, H.R., et al. (1997). Aggrecan degradation in human cartilage. Evidence for both matrix metalloproteinase and aggrecanase activity in normal, osteoarthritic, and rheumatoid joints. J. Clin. Invest 100, 93-106.

Le, L.T., Swingler, T.E., and Clark, I.M. (2013). Review: the role of microRNAs in osteoarthritis and chondrogenesis. Arthritis Rheum. 65, 1963-1974.

Lehar, J., Krueger, A.S., Avery, W., Heilbut, A.M., Johansen, L.M., Price, E.R., Rickles, R.J., Short, G.F., 3rd, Staunton, J.E., Jin, X., et al. (2009). Synergistic drug combinations tend to improve therapeutically relevant selectivity. Nat. Biotechnol. 27, 659-666.

Lewis, B.P., Burge, C.B., and Bartel, D.P. (2005). Conserved seed pairing, often flanked by adenosines, indicates that thousands of human genes are microRNA targets. Cell 120,15-20.

Matsukawa, T., Sakai, T., Yonezawa, T., Hiraiwa, H., Hamada, T., Nakashima, M., Ono, Y., Ishizuka, S., Nakahara, H., Lotz, M.K., et al. (2013). MicroRNA-125b regulates the expression of aggrecanase-1 (ADAMTS-4) in human osteoarthritic chondrocytes. Arthritis Res. Ther. 15, R28.

Maurer, B., Stanczyk, J., Jungel, A., Akhmetshina, A., Trenkmann, M., Brock, M., Kowal-Bielecka, O., Gay, R.E., Michel, B.A., Distler, J.H., et al. (2010). MicroRNA-29, a key regulator of collagen expression in systemic sclerosis. Arthritis Rheum. 62, 1733-1743.

Miyaki, S., and Asahara, H. (2012). Macro view of microRNA function in osteoarthritis. Nat. Rev. Rheumatol. 8, 543-552.

Miyaki, S., Sato, T., Inoue, A., Otsuki, S., Ito, Y., Yokoyama, S., Kato, Y., Takemoto, F., Nakasa, T., Yamashita, S., et al. (2010). MicroRNA-140 plays dual roles in both cartilage development and homeostasis. Genes Dev. 24, 1173-1185.

Murray, D.H., Bush, P.G., Brenkel, I.J., and Hall, A.C. (2010). Abnormal human chondrocyte morphology is related to increased levels of cell-associated IL-1beta and disruption to pericellular collagen type VI. J. Orthop. Res. 28, 1507-1514.

Noguchi, S., Yasui, Y., Iwasaki, J., Kumazaki, M., Yamada, N., Naito, S., and Akao, Y. (2013). Replacement treatment with microRNA143 and -145 induces synergistic inhibition of the growth of human bladder cancer cells by regulating PI3K/Akt and MAPK signaling pathways. Cancer Lett. 328, 353-361.

Park, J.K., Lee, E.J., Esau, C., and Schmittgen, T.D. (2009). Antisense inhibition of microRNA-21 or -221 arrests cell cycle, induces apoptosis, and sensitizes the effects of gemcitabine in pancreatic adenocarcinoma. Pancreas 38, e190-199.
Park, S.J., Cheon, E.J., Lee, M.H., and Kim, H.A. (2013). MicroRNA-127-5p regulates matrix metalloproteinase 13 expression and interleukin-1beta-induced catabolic effects in human chondrocytes. Arthritis Rheum. 65, 3141-3152.

Pencheva, N., Tran, H., Buss, C., Huh, D., Drobnjak, M., Busam, K., and Tavazoie, S.F. (2012). Convergent multi-miRNA targeting of ApoE drives LRP1/LRP8-dependent melanoma metastasis and angiogenesis. Cell 151, 1068-1082.

Salvat, C., Pigenet, A., Humbert, L., Berenbaum, F., and Thirion, S. (2005). Immature murine articular chondrocytes in primary culture: a new tool for investigating cartilage. Osteoarthritis Cartilage 13, 243-249.

Santini, P., Politi, L., Vedova, P.D., Scandurra, R., and Scotto d'Abusco, A. (2014). The inflammatory circuitry of miR-149 as a pathological mechanism in osteoarthritis. Rheumatol. Int. 34, 711-716.

Small, E.M., and Olson, E.N. (2011) Pervasive roles of microRNAs in cardiovascular biology. Nature 469, 336-342.

Song, J., Lee, M., Kim, D., Han, J., Chun, C.H., and Jin, E.J. (2013) MicroRNA-181b regulates articular chondrocytes differentiation and cartilage integrity. Biochem Biophys Res. Commun. 431, 210-214.

Swingler, T.E., Wheeler, G., Carmont, V., Elliott, H.R., Barter, M.J., Abu-Elmagd, M., Donell, S.T., Boot-Handford, R.P., Hajihosseini, M.K., Munsterberg, A., et al. (2012). The expression and function of microRNAs in chondrogenesis and osteoarthritis. Arthritis Rheum. 64, 1909-1919.

Wang, B., Komers, R., Carew, R., Winbanks, C.E., Xu, B., HermanEdelstein, M., Koh, P., Thomas, M., Jandeleit-Dahm, K., Gregorevic, P., et al. (2012). Suppression of microRNA-29 expression by TGF-beta1 promotes collagen expression and renal fibrosis. J. Am. Soc. Nephrol. 23, 252-265.

Wang, M., Sampson, E.R., Jin, H., Li, J., Ke, Q.H., Im, H.J., and Chen, D. (2013). MMP13 is a critical target gene during the progression of osteoarthritis. Arthritis. Res. Ther. 15, R5.

Xu, J., Li, C.X., Li, Y.S., Lv, J.Y., Ma, Y., Shao, T.T., Xu, L.D., Wang, Y.Y., Du, L., Zhang, Y.P., et al. (2011). MiRNA-miRNA synergistic network: construction via co-regulating functional modules and disease miRNA topological features. Nucleic Acids Res. 39, 825836.

Yamasaki, K., Nakasa, T., Miyaki, S., Ishikawa, M., Deie, M., Adachi, N., Yasunaga, Y., Asahara, H., and Ochi, M. (2009). Expression of MicroRNA-146a in osteoarthritis cartilage. Arthritis Rheum 60, 1035-1041.

Zhang, R., Ma, J., and Yao, J. (2013). Molecular mechanisms of the cartilage-specific microRNA-140 in osteoarthritis. Inflamm. Res. 62, 871-877.

Zhu, W., Zhao, Y., Xu, Y., Sun, Y., Wang, Z., Yuan, W., and Du, Z. (2013). Dissection of protein interactomics highlights microRNA synergy. PLoS One 8, e63342. 